\title{
Application of improved ant colony algorithm in vehicle scheduling
}

\section{problem}

\author{
Wang Jinguo ${ }^{1, a}$, Wang $\mathrm{Na}^{2, \mathrm{~b}^{*}}$ (corresponding author),Ma Haichun ${ }^{3, \mathrm{c}}$ \\ ${ }^{1}$ Department of Urology, the First Hospital of Jilin University, China \\ ${ }^{2}$ Department of Anesthesiology, the First Hospital of Jilin University, China \\ ${ }^{3}$ Department of Anesthesiology, the First Hospital of Jilin University, China

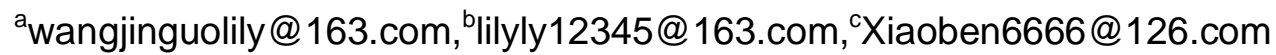

Keywords: Vehicle Scheduling. Ant Colony Algorithm. Pheromone.

Abstract. In this paper, the ant colony algorithms is studied, and improve the shortcomings of the algorithm, And the improved algorithm is introduced into the field of logistics transportation. Aiming at the complexity and uncertainty of logistics transportation vehicle scheduling problem, a new algorithm is designed. The experimental results show that the improved algorithm can choose the transport route, speed up the transportation speed, improve the service quality, reduce the transportation cost and increase economic benefits.

\section{Introduction}

The vehicle scheduling problem was first proposed by Dantzing and Ramser in 1959. Because of this problem is involved in many subject, the theoretical abstraction of many practical problems can be attributed to this problem; it has a broad application prospect. It has always been the research hotpots in the field of operations research and combination optimization[1].

Vehicle optimal scheduling problem is classified according to the relative importance of the spatial and temporal characteristics of the problem[2]. Modern logistics transportation vehicle scheduling process is complicated, and the existing mathematical methods in solving this problem is not perfect, the lack of scientific theory for guidance. To solve these problems, it is often required to make decisions and judgments with the heuristic method, and the optimal solution of the overall optimum, the total cost and the total benefit of the transportation system[3].

In China, with the rise of the logistics industry, we put forward the comprehensive requirements of economic, accuracy and flexibility for the transportation of goods. Therefore, the research work in this area is being carried out in a large scale. Scholars study the form, analysis, and model and solving method of vehicle scheduling, which have practical significance and application value to the establishment of modern logistics transportation[4].

\section{Vehicle scheduling problem model}

In order to establishing the model facilitate, we make the following assumptions. Vehicle weight is known. The vehicles only can unloading and no loading at each service point. The demand point of distribution task are limited by time window. Time window range is set according to the needs of each demand point. The average speed of the vehicle is known, the distance is proportional to the time. Under these assumptions, the construction of the mathematical model is as follows: 
$Z=\min \sum_{k=1}^{k}\left[\sum_{i=1}^{S} l_{D^{i-1} D^{i}}+l_{D S}\right] \operatorname{sign}(S)$

and

$\operatorname{sign}(S)=\left\{\begin{array}{c}0 \quad \text { if } \quad S>=1 \text {, show that the car has participated in the distribution } \\ 1 \quad \text { if } S=0 \text {, show that the car doesn't participated in the distribution }\end{array}\right.$ (2) Constrain $\mathrm{t}$ conditions are as follow:

$\sum_{i=1}^{S} G_{D^{i}}<=T G$ in which, $D \neq 0$

$\sum_{i=1}^{S} l_{D^{i-1} D^{i}}+l_{D S}<=T l$ in which, $S \neq 0$

$0<=S<=N$ and $\sum_{i=1}^{i} S=N$

\section{Improvement of ant colony algorithm}

The basic ant colony algorithm has a strong ability to find optimal solutions and it can converge to a certain subset of the solution space faster than other algorithms[5]. However, when the problem size is large or the size of the population is large, the algorithm's calculation is large, and the basic ant colony algorithm often has two problems. it is easy to fall into local optimum, namely the search to a certain extent; all individuals find solution are basically the same. It cannot further search the solution space. It maybe not finds the global optimal solution. The other is that the time of convergence to the global optimal solution is longer. The solution results are shakes between the local optimal solution and the global optimal solution[6]. In order to solve the problem, this paper makes the following improvements on ant colony algorithm.

Pheromone updates rule improvement. According to the real world, it is the higher the concentration of pheromone, the more volatile, and the lower the concentration of pheromone, and the lower volatile. It can prevent that the pheromone is increased infinite on some path and at the same time, others decreased to the zero. This will reduce the probable that the solution falls into the local optimum. In such a case, the volatility factor is changed from constant to function with as a variable. Therefore, the following global update rules are used:

$\tau_{i j}=(1-\rho) \tau_{i j}+\sum_{\alpha=1}^{m} \Delta \tau_{i j}^{\alpha}$

In which, $\Delta \tau_{i j}^{\alpha}(t)=\left(C_{\max }^{\alpha}\right)^{-1}$.

It presents the pheromone how the ant released. The value of $C_{\max }$ is inversely proportional to the solution of the ant colony. The $\rho$ is pheromone evaporation, it between $(0,1)$. The $\mathrm{C}$ is a constant.

Update strategy of the $C$. The update coefficient $C$ will affect the convergence rate of the algorithm. If the $\mathrm{C}$ larger, it will make the algorithm converge to the local minimum. Contrary too small, it will affect the convergence speed of the algorithm. With the problem increasing, in order to speed up the convergence rate and increase the search range, we use different update coefficient $\mathrm{C}$ 
in different stages. At the same time, the number of different paths is selected at different stages of the cycle. In the initial time, the smaller coefficient $\mathrm{C}$ is used. In this way, the pheromone on each path is changed slowly, and in the search process the probability of choosing the other paths is relatively large. At the end of the cycle, we use the large update coefficient, and choose the shortest path in the loop as the update path. This is only the enhanced pheromone of the optimal path can correctly guide the ants' next search. So as to avoid a large number of invalid searches, significantly improve the search efficiency.

\section{Algorithm simulation and results analysis}

There are seven points of vehicle routing problem, each task point coordinates and freight are shown in table 1.

Table1.The delivery point coordinates and freight

\begin{tabular}{|c|c|c|c|c|c|c|c|c|}
\hline & 0 & 1 & 2 & 3 & 4 & 5 & 6 & 7 \\
\hline coordinate & $(15,40)$ & $(20,50)$ & $(15,10)$ & $(28,12)$ & $(42,65)$ & $(69,80)$ & $(79,45)$ & $(90,15)$ \\
\hline freight & & 1 & 0.5 & 0.3 & 0.6 & 0.4 & 0.2 & 0.3 \\
\hline
\end{tabular}

0 is a center warehouse. Assumption vehicle capacity is $q=1$. Ant colony algorithm of parameter

Settings are as follows: $\alpha=0.8, \rho=0.75$. The max number of iterations is $\mathrm{NC}=50$. The number of ants is 15 . If the $\mathrm{NC}<10$, the constant $\mathrm{C}$ is 0.8 . If $\mathrm{NC}<=10$ AND $\mathrm{NC}<30$, the constant $\mathrm{C}$ is 0.3 , else the constant $\mathrm{C}$ is 0.9 .

In the process of ant colony algorithm running, all found the optimal logistics distribution path: 268.92. At the same time spent less time, less number of iterations. The shortest path algorithm is shown in figure 1.

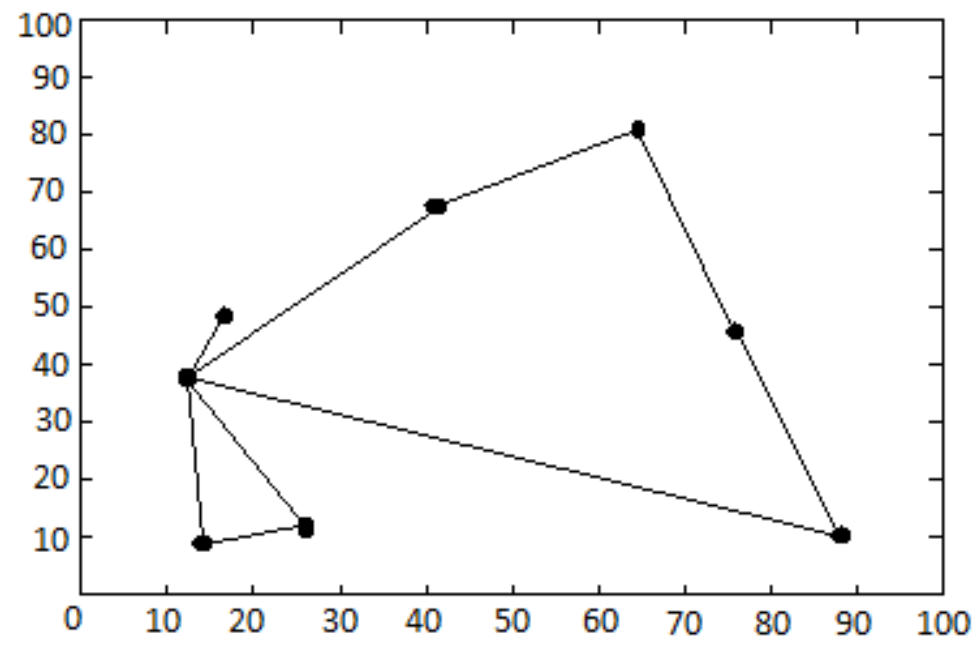

Fig1.the optimal distribution path graph 


\section{Conclusion}

In this paper, we propose a improved ant colony algorithm to solve the vehicle scheduling problem. We study the general ant colony algorithm is easy to stagnation and premature convergence, put forward from the pheromone updating rule, such as updating coefficient and update strategy for the changes. Experiments prove that the scheme effectively improves the algorithm convergence speed, expand the search space of the algorithm.

\section{References}

[1] Gambardella L M, Dorigo M. Ant - Q: a reinforcement learning approach to the traveling salesman Problem. Proceedings of the 12th Intentional Conference on Machine Learning, 1995,252 $-260$.

[2] Shin Watanabe,Atsushi Tero,Atsuko Takamatsu,Toshiyuki Nakagaki. Traffic optimization in railroad networks using an algorithm mimicking an amoeba-like organism, Physarum plasmodium[J]. BioSystems . 2011 (3).

[3] Christian Blum,Mateu Yábar Vallès,Maria J. Blesa. An ant colony optimization algorithm for DNA sequencing by hybridization[J]. Computers and Operations Research . 2007 (11).

[4]Atsushi Tero,Ryo Kobayashi,Toshiyuki Nakagaki. A mathematical model for adaptive transport network in path finding by true slime mold[J]. Journal of Theoretical Biology . 2006 (4)。

[5] Nan Zhao,Zhilu Wu,Yaqin Zhao,Taifan Quan. Ant colony optimization algorithm with mutation mechanism and its applications[J]. Expert Systems With Applications . 2009 (7)

[6] Guenther Fuellerer,Karl F. Doerner,Richard F. Hartl,Manuel Iori. Ant colony optimization for the two-dimensional loading vehicle routing problem[J]. Computers and Operations Research . 2007 (3) . 